\title{
Increased velocity and induction of chemotactic response in mouse spermatozoa by follicular and oviductal fluids
}

\author{
R. G. Oliveira, L. Tomasi, R. A. Rovasio* and L. C. Giojalas \\ Cátedra de Biología Celular, Facultad de Ciencias Exactas, Físicas y Naturales, \\ Universidad Nacional de Córdoba, Av. Vélez Sarsfield 299, (5000) Córdoba, Argentina
}

\begin{abstract}
The dynamic parameters of mouse sperm cells exposed to follicular and oviductal fluids were assessed. Spermatozoa were tracked on a chemotactic Zigmond chamber and recorded using a videomicroscopy system. The results were evaluated with computer-supported image analysis. Follicular fluid at a dilution of $10^{4}$ markedly increased the proportion of spermatozoa with high velocity, and stimulated chemotactic behaviour. The highest velocities were observed in sperm cells exposed to oviductal fluid, and a greater proportion of these cells had high velocity compared with those exposed to follicular fluid. Chemotaxis was induced in spermatozoa exposed to oviductal fluid at dilutions of $10^{-3}$ and $10^{-5}$. These results suggest the presence of temporal subpopulations of responsive spermatozoa, considering the distance travelled towards both follicular and oviductal fluids and the proportion of sperm cells migrating towards the gradient in the highest distance ranges. This is the first report on the effect of isolated follicular and oviductal fluids on dynamic parameters and chemotaxis of mouse spermatozoa. The findings support previous work showing that the motility and directionality of mouse sperm cells is increased by factors in the microenvironment of the egg. Although the significance of these factors in vivo is unknown, it is possible that there is a relay mechanism involving sequential activity of both oviductal and follicular fluids to direct the male gametes towards the egg.
\end{abstract}

\section{Introduction}

The oviductal isthmus of several mammalian species acts as a storage site in which quiescent sperm cells accumulate and remain attached temporally (Hunter, 1987; Katz et al., 1989), and capacitation probably occurs (First and Parrish, 1987). Eisenbach (1995) proposed that only capacitated spermatozoa can detach from the oviductal epithelium and swim to the egg in a chemotactically directed manner. However, it is known that at ovulation, sperm function can be modified by the composition of fluids present in the female reproductive system. It has been shown that human follicular fluid enhances sperm cell motility and velocity (Mendoza and Tesarik, 1990; Falcone $e t$ al., 1991; Fetterolf $e t$ al., 1994), and elicits a sperm chemotactic response (Ralt et al., 1991, 1994; Eisenbach and Ralt, 1992; Eisenbach and Tur-Kaspa, 1994). Oviductal fluid can also stimulate human sperm cell motility (Zhu et al., 1994). However, chemotaxis has not yet been demonstrated with oviductal fluid.

In a recent study, the velocity of spermatozoa from the mouse epididymis was shown to increase and the spermatozoa move towards a gradient of crude extract from the microenvironment of the egg (Giojalas and Rovasio,

${ }^{*}$ Correspondence.

Received 9 March 1998.
1998). The aim of this work was to evaluate the dynamic parameters and the chemotactic response of mouse sperm cells exposed to follicular and oviductal fluids.

\section{Materials and Methods}

All animals were handled in accordance with UK legal requirements and the Guide for the Care and Use of Laboratory Animals (US Department of Health and Human Services, 1985).

\section{Preparation of spermatozoa}

Three-month-old inbred C57-BL/6 male mice were killed by cervical dislocation. Cauda epididymides were removed and placed in a plastic dish containing $400 \mu \mathrm{l}$ Dulbecco's modified Eagle's medium (DMEM; Sigma, St Louis, MO), with $0.1 \%$ polyvinyl alcohol (Sigma, St Louis, MO) and $20 \mathrm{mmol}$ Hepes $1^{-1}$ (Sigma, St Louis, MO), pH 7.4. The epididymides were cut into several fragments and incubated at $37^{\circ} \mathrm{C}$ for $10 \mathrm{~min}$ to allow the spermatozoa to disperse. The sperm cell suspension was collected in a plastic Eppendorf microtube; DMEM was added to achieve a volume of $1 \mathrm{ml}$; and the cells were distributed homogeneously by slowly 
rolling the tube between the hands for $5 \mathrm{~min}$. After incubation at $37^{\circ} \mathrm{C}$ for $90 \mathrm{~min}$, the upper $200 \mu \mathrm{l}$ of the cell suspension was removed and diluted 1:1 with DMEM. Controls without protein were introduced. The dynamic behaviour of mouse spermatozoa is the same with or without the addition of protein (Giojalas and Rovasio, 1998).

\section{Isolation of follicular fluid}

Superovulation was induced in 3-month-old inbred C57$\mathrm{BL} / 6$ female mice by intraperitoneal injection of $7.5 \mathrm{iu} \mathrm{FSH}$ (Serono, Buenos Aires) and, after $48 \mathrm{~h}$, intraperitoneal injection of 20 iu hCG (Elea, Buenos Aires). After $12 \mathrm{~h}$, and before ovulation, the females were killed by cervical dislocation. Ovaries were placed in wells of a Terasaki microplate (Becton Dickinson Co., OxnardCockeysville, MD) with $10 \mu \mathrm{l}$ DMEM. Mature follicles were punctured with a tungsten microneedle under a stereoscopic microscope and the pool of fluid recovered was centrifuged at $5600 \mathrm{~g}$ for $10 \mathrm{~min}$. The amount of follicular fluid in the supernatant was estimated as the protein concentration (Bradford, 1976). The supernatant from several females was pooled and stored at $-20^{\circ} \mathrm{C}$. For the experiments, the follicular fluid was diluted $10^{-3}, 10^{-4}$, and $10^{-5}$ with DMEM, corresponding to protein concentrations of 100,10 and $1 \mu \mathrm{g} \mathrm{ml}^{-1}$, respectively.

\section{Isolation of oviductal fluid}

Three-month-old inbred C57-BL/6 female mice were anaesthetized with ether (Rodia, Buenos Aires) and the dorsolateral zones were surgically opened. Oviducts were ligated at the uterine and ovarian ends with absorbable suture thread to collect the oviductal fluid. The mice were maintained under standard breeding conditions for 15 days and then killed by cervical dislocation. The oviducts were recovered and oviductal fluid was aspirated with a capillary needle under a microscope. The fluid was pooled and centrifuged at $5600 \mathrm{~g}$ for $10 \mathrm{~min}$. Supernatant containing the oviductal fluid was stored at $-20^{\circ} \mathrm{C}$. For the experiments, the oviductal fluid was diluted $10^{-3}, 10^{-4}$ and $10^{-5}$ with DMEM, corresponding to protein concentrations of 100,10 and $1 \mu \mathrm{g}$ $\mathrm{ml}^{-1}$, respectively).

\section{Cell motility recording and evaluation of dynamic parameters}

Experiments were performed in a chemotactic Zigmond chamber (Zigmond, 1977), consisting of an acrylic slide with two compartments separated by a $1 \mathrm{~mm}$ wall (Fig. 1). The chamber was closed with a coverslip treated with $1 \%(\mathrm{w} / \mathrm{v})$ nitrocellulose in alcohol:ether (1:1) to avoid the 'sticking-toglass' phenomenon (Chapeau and Gagnon, 1987), and was sealed with silicon oil. One compartment was filled with follicular fluid, oviductal fluid or DMEM (control group), and the other with the sperm cell suspension, thus forming a concentration gradient between the compartments. The spermatozoa were exposed to the follicular fluid, oviductal

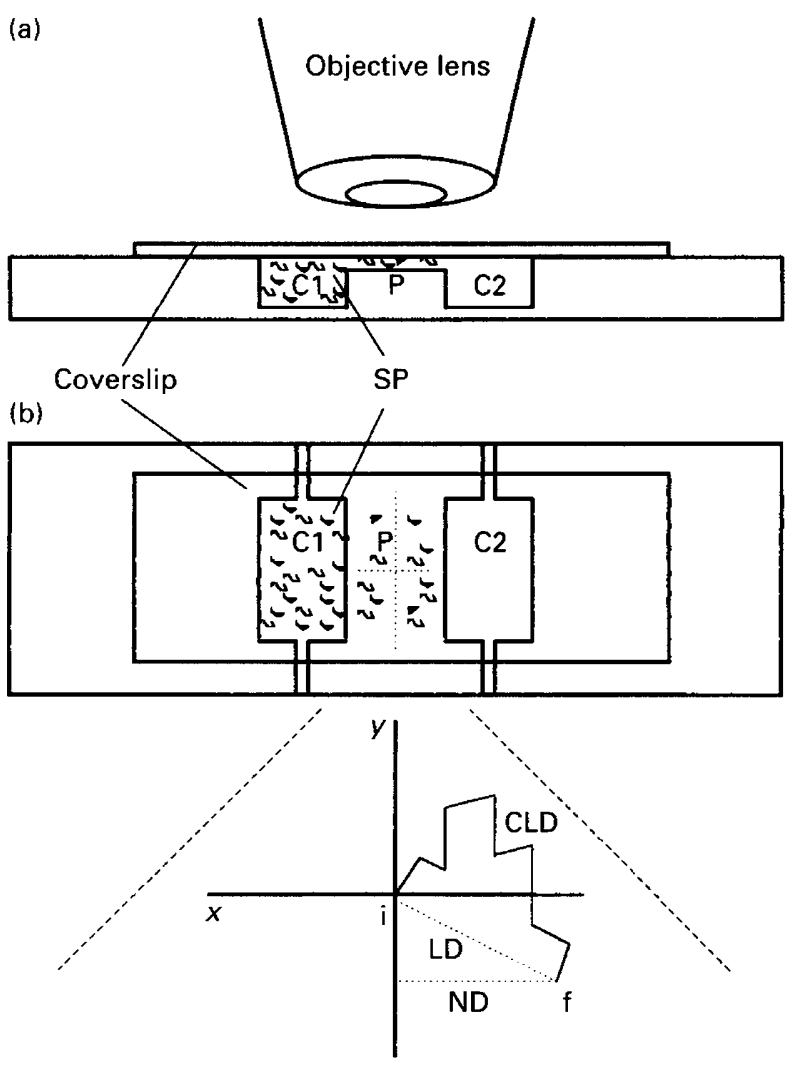

Fig. 1. Experimental system used for determination of the dynamic parameters of mouse spermatozoa (SP). (a) Vertical section and (b) superior view of the Zigmond chamber, with the two compartments separated by a central partition $(\mathrm{P})$. $\mathrm{C} 1$, compartment containing sperm suspension; $\mathrm{C} 2$, compartment containing follicular or oviductal fluid or culture medium (control). A projection of the $x-y$ co-ordinates system and a cell trajectory with related dynamic parameters is shown at the bottom of the figure. $i$, starting point of cell trajectory; $f$, final point of the cell trajectory; CLD, curvilinear distance, $L D$, linear distance; ND, net displacement.

fluid or DMEM at $37^{\circ} \mathrm{C}$ for $10 \mathrm{~min}$. The procedure for sealing the chamber and the short duration of the assay help to maintain stable atmospheric conditions in the system. The movement of the spermatozoa was tracked and recorded under a phase-contrast microscope at $\times 100$ magnification for periods of $10 \mathrm{~s}$, focusing on the partition between the compartments. The sperm cell tracks were then transferred to an acetate sheet attached to the monitor screen and were analysed at $60 \mathrm{~Hz}$ for $3 \mathrm{~s}$ in real time using the semiautomatic objective method (Marin et al., 1995; Rovasio et al., 1995). Each experiment was conducted in triplicate with sperm samples from different males and at least 150 spermatozoa were analysed per sample.

The cell motility parameters determined were: curvilinear velocity (VCL), the total distance travelled per unit time; linear velocity (VSL), the distance between the initial and final points of the track per unit time; linearity (LIN), a dimensionless number obtained as the quotient between VSL and VCL that expresses the straight direction of the track (Hinting et al., 1988); and chemotaxis, the net distance 
parallel to the gradient travelled by the spermatozoon towards the attractant. For this purpose, an axial Cartesian system was drawn on the acetate sheet, in which the $y$ axis represented the Zigmond chamber partition between the compartments, and the $x$ axis represented the concentration gradient (Fig. 1). The start point of each track was drawn in the centre of the Cartesian system, and the final point at the corresponding left- or right-hand side. The net distance travelled by each spermatozoon was expressed as the distance between both ends of the track after transporting them over the $x$ axis. The final value of this parameter was calculated as the distance spermatozoa migrated towards the attractant, minus the mean distance corresponding to the cells migrating against the gradient.

\section{Statistical analysis}

The Student's $t$ test was used to compare the mean values among treatments and the $Z$ test was used to evaluate differences among percentages (Montgomery, 1991) using the SigmaStat software (Jandel Scientific, San Rafael, CA).

\section{Results}

\section{Effects of follicular fluid}

At dilutions of $10^{-3}$ and $10^{-4}$, follicular fluid significantly increased the spermatozoa VCL, VSL and LIN values compared with the control group $(P<0.001)$, and at $10^{-5}$, VSL and LIN values were markedly greater than in controls $(P<0.001)$ (Table 1). Analysis of the proportion of sperm cells with different VCL ranges revealed a greater proportion of spermatozoa in the range $231-350 \mu \mathrm{m} \mathrm{s}^{-1}$ at $10^{-3}$ and $10^{-4}$ dilutions of follicular fluid $(P<0.01)$, whereas in the greatest VCL ranges, the proportion of sperm cells was low and similar in all the experimental groups (Fig. 2). The chemotactic behaviour of spermatozoa was significantly greater in the presence of follicular fluid at a dilution of $10^{-4}$ (Fig. 3). In all dilutions of follicular fluid, a few spermatozoa moved a short distance against the gradient, while others moved a long distance towards the follicular fluid gradient
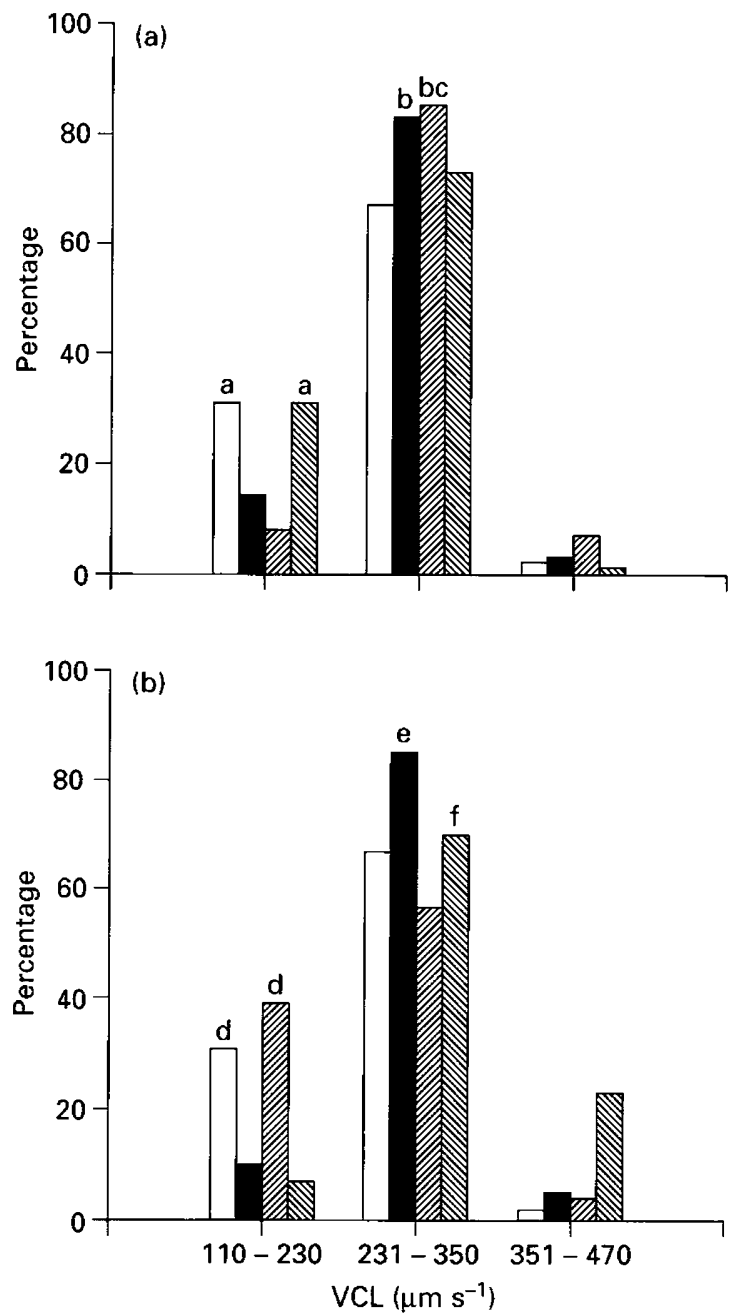

Fig. 2. Frequency distribution of mouse spermatozoa according to different curvilinear velocity (VCL) ranges after exposure to (a) follicular fluid or (b) oviductal fluid at a dilution of $(\mathbf{\square}) 10^{-3}$, (ख) $10^{-4}$ and $\left.(\mathbb{Q}) 10^{-5} ; \square\right)$ control. Letters indicate significant differences with respect to: a, follicular fluid at $10^{-3}$ and $10^{-4}(P<0.001)$; $b$, controls and follicular fluid at $10^{-5}(P<0.01) ; c$, oviductal fluid at $10^{-4}$ $(P<0.001)$; d, oviductal fluid at $10^{-3}$ and $10^{-5}(P<0.001)$; e, controls and oviductal fluid at $10^{-4}$ and $10^{-5}(P<0.01) ; \mathrm{f}$, oviductal fluid at $10^{-4}$ $(P<0.001)$.

Table 1. Dynamic parameters of mouse spermatozoa exposed to follicular or oviductal fluid

\begin{tabular}{|c|c|c|c|c|}
\hline & Dilution & $\operatorname{VCL}\left(\mu \mathrm{m} \mathrm{s}^{-1}\right)$ & $\operatorname{VSL}\left(\mu \mathrm{m} \mathrm{s}^{-1}\right)$ & LIN \\
\hline Control & - & $258.5 \pm 4.02$ & $62.0 \pm 1.25$ & $0.25 \pm 0.01$ \\
\hline \multirow[t]{3}{*}{ Follicular fluid } & $10^{-3}$ & $276.0 \pm 3.46^{\mathrm{ab}}$ & $76.3 \pm 1.59^{\mathrm{a}}$ & $0.28 \pm 0.01^{\mathrm{a}}$ \\
\hline & $10^{-4}$ & $280.8 \pm 3.25^{\mathrm{abf}}$ & $79.9 \pm 1.30^{a f}$ & $0.29 \pm 0.005^{\mathrm{af}}$ \\
\hline & $10^{-5}$ & $254.3 \pm 2.79(\mathrm{~ns})$ & $81.3 \pm 0.98^{\mathrm{ac}}$ & $0.33 \pm 0.005^{\mathrm{ad}}$ \\
\hline \multirow[t]{3}{*}{ Oviductal fluid } & $10^{-3}$ & $280.2 \pm 3.53^{\text {ae }}$ & $73.7 \pm 1.76^{\mathrm{ae}}$ & $0.27 \pm 0.01^{\mathrm{a}}$ \\
\hline & $10^{-4}$ & $246.4 \pm 3.84(\mathrm{~ns})$ & $61.9 \pm 1.61(\mathrm{~ns})$ & $0.26 \pm 0.01(\mathrm{~ns})$ \\
\hline & $10^{-5}$ & $302.1 \pm 4.7^{\text {adg }}$ & $83.7 \pm 1.95^{\mathrm{ad}}$ & $0.28 \pm 0.01^{\text {aef }}$ \\
\hline
\end{tabular}

Values are means $\pm \mathrm{SE}$.

VCL, curvilinear velocity; VSL, linear velocity; $L I N$, linearity.

Superscripts indicate significant differences with respect to: a, control group $(P<0.001)$; b, follicular fluid at $10^{-5}(P<0.0001)$; c, follicular fluid at $10^{-3}(P<0.01)$; $\mathrm{d}$, the corresponding follicular fluid or oviductal fluid at $10^{-3}$ and $10^{-4}(P<0.0001)$; e, oviductal fluid at $10^{-4}(P<0.01)$; f, oviductal fluid at $10^{-4}(P<0.0001)$; g, follicular fluid at $10^{-5}(P<0.0001)$. ns, not significant. 


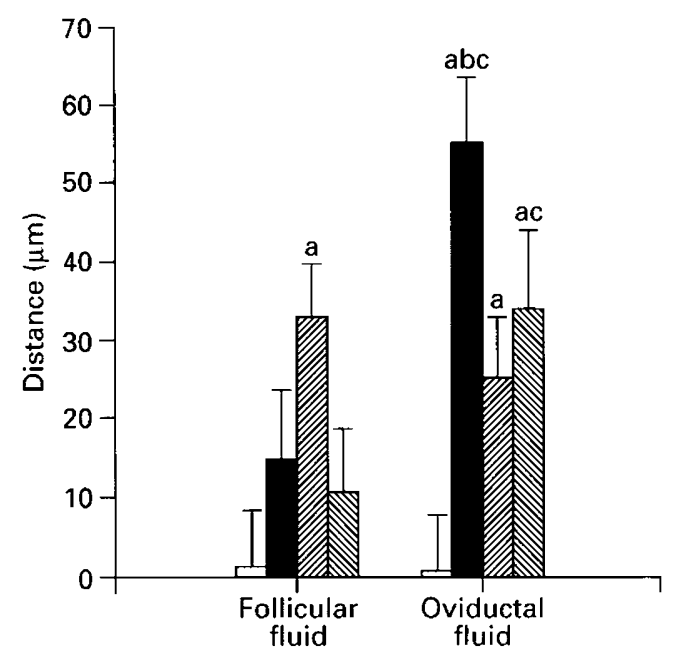

Fig. 3. Chemotactic behaviour of mouse spermatozoa expressed as the distance parallel to the gradient travelled towards follicular or oviductal fluid. The fluids were used at dilutions of $(\square) 10^{-3},(\mathscr{Z}) 10^{-4}$ and $\left.(\mathbb{Q}) 10^{-5} ; \square\right)$ control. Letters indicate significant differences with respect to: a, control $(P<0.01)$; b, oviductal fluid at $10^{-4}(P<0.01)$; c, follicular fluid $(P<0.001)$. Values are means \pm SEM.

(Fig. 4). In the control group, there was a small proportion of sperm cells in all the distance ranges, indicating that the spermatozoa were distributed randomly (Fig. 4).

\section{Effects of oviductal fluid}

A significant increase in the dynamic parameters was found when the sperm cells were exposed to oviductal fluid at dilutions of $10^{-3}$ and $10^{-5}(P<0.0001)$ (Table 1$)$. However, a greater proportion of spermatozoa travelled in the middle range of VCL values $\left(231-350 \mu \mathrm{m} \mathrm{s}^{-1}\right)$ in the presence of oviductal fluid at a dilution of $10^{-3}(P<0.001)$ (Fig. 2). Furthermore, in all three dilutions of oviductal fluid, sperm cells showed positive chemotactic behaviour, travelling a greater distance towards the oviductal fluid gradient $(P<0.01)$ than controls (Fig. 3). The highest displacement value was observed in the group of sperm cells exposed to oviductal fluid at a dilution of $10^{-3}(P<0.001)$ (Fig. 3). In addition, spermatozoa exposed to oviductal fluid at all three dilutions travelled a greater distance towards the oviductal fluid gradient, in comparison with the short distance travelled against the oviductal fluid gradient. In the control group, a small and constant proportion of sperm cells was present in both left- and right-hand fields and the directionality was random in all the distance ranges studied (Fig. 4).

\section{Discussion}

The results of this study show that both oviductal and follicular fluids enhance the dynamic parameters and induce a chemotactic response in epididymal mouse spermatozoa at a dilution of $10^{-3}$ and $10^{-5}$ for oviductal fluid and $10^{-4}$ for follicular fluid. Although oviductal fluid induced more sperm cells to move a greater distance towards the
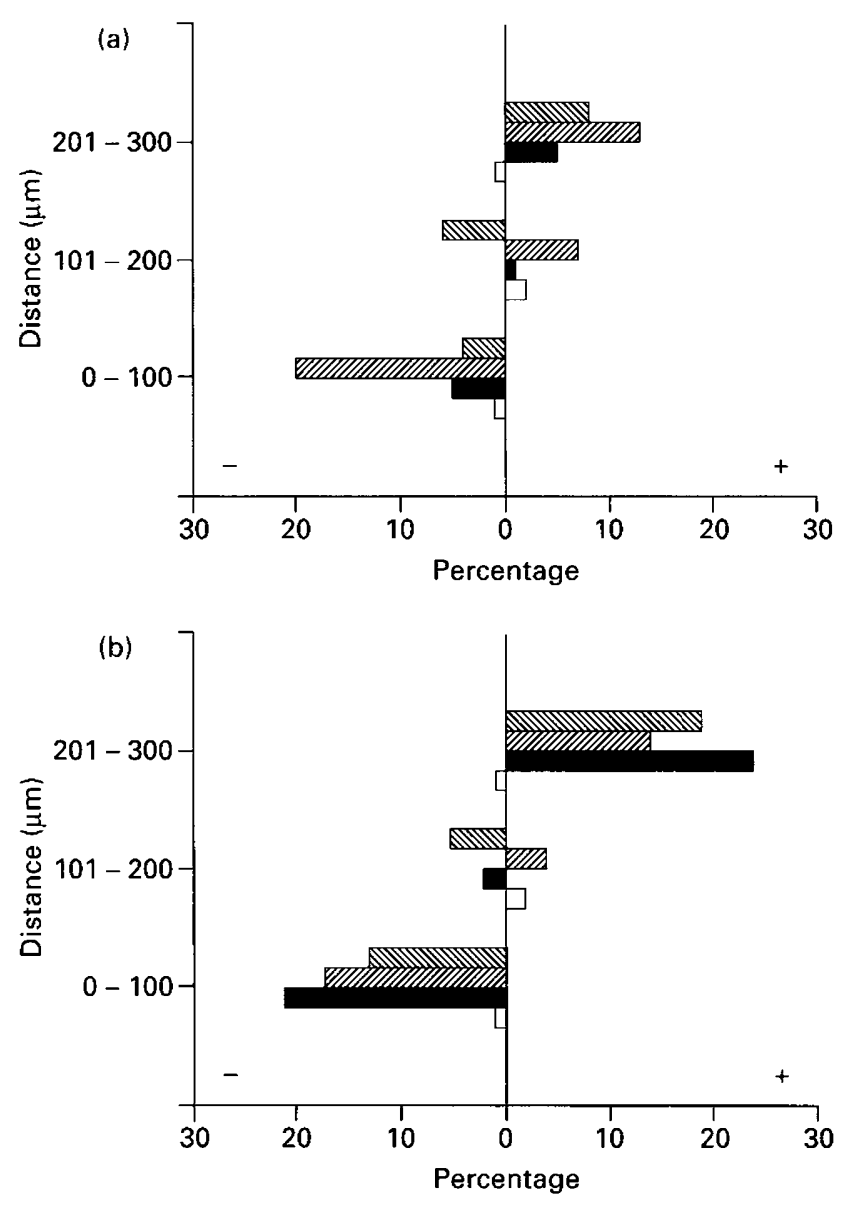

Fig. 4. Frequency distribution of mouse spermatozoa movements, expressed as the difference between the percentage of spermatozoa moving towards and away from (a) follicular and (b) oviductal fluid, according to different ranges of distance parallel to the gradient of the fluids. The fluids were used at dilutions of $(\boldsymbol{D}) 10^{-3},(\mathbb{Z}) 10^{-4}$ and (ब) $10^{-5} ;(\square)$ control. + , Zigmond chamber side containing the test solution; -, Zigmond chamber side containing the sperm suspension.

chemotactic gradient, follicular fluid caused the greatest increase in the velocity of spermatozoa. These results are in agreement with those obtained in humans, in which both follicular and oviductal fluids modify several sperm functions, including velocity (Mendoza and Tesarik, 1990; Falcone et al., 1991; Fetterolf et al., 1994; Zhu et al., 1994), and follicular fluid exerts a chemotactic effect (Ralt et al., 1991, 1994; Eisenbach and Ralt, 1992; Cohen-Dayag et al., 1994; Eisenbach and Tur-Kaspa, 1994). Follicular fluid at a dilution of $10^{-4}$ had the greatest chemotactic response. This is in accordance with the study of Ralt et al. (1991) using human spermatozoa. The reason why the values obtained for oviductal fluid at dilutions of $10^{-3}$ and $10^{-5}$ were lower than those obtained at $10^{-4}$ remains to be elucidated. It is possible that follicular and oviductal fluids exert an effect at a narrow concentration range, as has been reported for other biological systems (Schlatterer and Malchow, 1993; Felder and Kam, 1994; Fukui and Inoué, 1997).

This is the first report on the effects of isolated follicular and oviductal fluids on the dynamic parameters and 
chemotaxis of mouse spermatozoa. The findings support previous work demonstrating the enhanced motility and directionality of mouse spermatozoa by factors in the microenvironment of the egg (Giojalas and Rovasio, 1998). The results suggest the presence of temporal subpopulations of responsive spermatozoa, considering the distance travelled towards both follicular and oviductal fluids, as well as the proportion of sperm cells migrating towards the gradient at the highest distance ranges.

The proportion of sperm cells showing the greatest chemotactic response to follicular fluid (about 13\%) is in agreement with previous studies in mice (Giojalas and Rovasio, 1998) and humans (Cohen-Dayag et al., 1994). A greater proportion of sperm cells $(24 \%)$ showed a marked chemotactic response in the presence of oviductal fluid, suggesting that the activity of oviductal factors increases dynamic behaviour.

During insemination in vivo, only a few thousand of the millions of spermatozoa inseminated reach the isthmus of the oviduct, and only a few reach the ampulla at fertilization (Suarez et al., 1990). In mice, as in other mammals, sperm cells entering the oviduct attach to the isthmus storage sites and reduce their movement (Suarez, 1987). At ovulation, a few spermatozoa recover their motility and swim from the reservoir (Hunter and Nichol, 1983; Hunter and Wilmut, 1984; Smith et al., 1987). It has been proposed that only capacitated spermatozoa are released from the isthmus and reach the site of fertilization by chemotactic translocation (Eisenbach, 1995). The results of the present study indicate that both female fluids enhance sperm cell motility and induce a chemotactic response, although the significance of this in vivo is unknown. A hypothesis involving a relay mechanism with two sequential steps is proposed to explain the results. When spermatozoa reach the oviduct, the oviductal fluid enhances their motility and directs them chemotactically towards the isthmus storage site. When capacitated spermatozoa detach from the reservoir, follicular fluid enhances their speed and directs the male gametes chemotactically towards the ampulla area to contact the egg. Further studies are required to test the rationale of this hypothesis.

This work was supported by grants from CONICET, CONICOR and SECyT-UNC (Argentina), and The Third World Academy of Sciences (TWAS). R. A. Rovasio and L. C. Giojalas are members of the researcher career scheme of the National Council of Scientific Research and Technology (CONICET). The authors are especially grateful to D. Abal for graphic design, and to M. González-Cremer for critical reading of the manuscript.

\section{References}

Bradford M (1976) A rapid and sensitive method for the quantitation of microgram quantities of protein utilizing the principle of protein-dye binding Analytical Biochemistry 72 248-254

Chapeau C and Gagnon C (1987) Nitrocellulose and polyvinyl coatings prevent sperm adhesion to glass without affecting the motility of intact and demembranated human spermatozoa Journal of Andrology 834-40

Cohen-Dayag A, Ralt D, Tur-Kaspa I, Manor M, Makler A, Dor J, Mashiach $\mathbf{S}$ and Eisenbach $\mathbf{M}$ (1994) Sequential acquisition of chemotactic responsiveness by human spermatozoa Biology of Reproduction 50 786-790

Eisenbach M (1995) Sperm changes enabling fertilization in mammals Current Opinion in Endocrinology and Diabetes 2 468-475
Eisenbach $M$ and Ralt D (1992) Pre-contact mammalian sperm-egg communication and its role in fertilization American Journal of Physiology $\mathbf{2 6 2}$ C1095-1101

Eisenbach M and Tur-Kaspa I (1994) Human sperm chemotaxis is not enigmatic anymore Fertility and Sterility 62 233-235

Falcone L, Soldati G, Piffaretti-Yanez A, Marchini M, Eppenberger $U$ and Balerna M (1991) Follicular fluid enhances sperm motility and velocity in vitro. Fertility and Sterility 55 619-623

Felder S and Kam Z (1994) Human neutrophil motility: time-dependent threedimensional shape and granule diffusion Cell Motility and the Cytoskeleton 28 285-302

Fetterolf PM, Sutherland CS, Josephy PD, Casper RF and Tyson JE (1994) Preliminary characterization of a factor in human follicular fluid that stimulates human spermatozoa motion Human Reproduction 9 1505-1511

First NL and Parrish JJ (1987) In vitro fertilization of ruminants Journal of Reproduction and Fertility Supplement 34 151-165

Fukui $Y$ and Inoué $S$ (1997) Amoeboid movement anchored by eupodia, new actin-rich knobby feet in Dictyostelium. Cell Motility and the Cytoskeleton 36 339-354

Giojalas LC and Rovasio RA (1998) Mouse spermatozoa modify their dynamic parameters and chemotactic response to factors from egg microenvironment International Journal of Andrology 21 201-206

Hinting A, Schoonjans F and Comhaire F (1988) Validation of a single-step procedure for the objective assessment of sperm motility characteristics International Journal of Andrology 11 277-278

Hunter RFH (1987) Human fertilization in vivo, with special reference to progression, storage and release of competent spermatozoa Human Reproduction 229-232

Hunter RFH and Nichol R (1983) Transport of spermatozoa in the sheep oviduct: preovulatory sequestering of cells in the caudal isthmus Journal of Experimental Zoology 228 121-128

Hunter RFH and Wilmut I (1984) Sperm transport in the cow: periovulatory redistribution of viable cells within the oviduct Reproduction Nutrition and Development 24 597-608

Katz DF, Drobnis EZ and Overstreet JW (1989) Factors regulating mammalian sperm migration through the female reproductive tract and oocyte vestments Gamete Research $22443-469$

Marín CI, Calamera JC and Rovasio RA (1995) Development of an objective, semiautomated method for assessment of sperm motility Andrologia 27 $115-119$

Mendoza C and Tesarik J (1990) Effect of follicular fluid on sperm movement characteristics Fertility and Sterility 54 1135-1139

Montgomery DC (1991) Design and Analysis of Experiments 3rd Edn John Wiley \& Sons, New York

Ralt D, Goldenberg M, Fetterolf P, Thompson D, Dox J, Mashiach S, Garbers DL and Eisenbach $M$ (1991) Sperm attraction of follicular factor(s) correlates with human egg fertilizability Proceedings National Academy of Sciences USA 88 2840-2844

Ralt D, Manor M, Cohen-Dayag A, Tur-Kaspa I, Makler A, Yuli I, Dor J, Blumberg S, Mashiach S and Eisenbach M (1994) Chemotaxis and chemokinesis of human spermatozoa to follicular factors Biology of Reproduction 50 774-785

Rovasio RA, Giojalas L and Marín C (1995) Three seconds is the optimal tracking time to distinguish different mouse sperm patterns Comunicaciones Biológicas 12 313-320

Schlatterer C and Malchow D (1993) Intracellular guanosine-5'-O-(3thiotriphosphate) blocks chemotactic motility of Dictyostelium discoideum amoebae Cell Motility and the Cytoskeleton 25 298-307

Smith TT, Koyangi F and Yanagimachi R (1987) Distribution and number of spermatozoa in the oviduct of the golden hamster after mating and artificial insemination Biology of Reproduction 37 225-234

Suarez SS (1987) Sperm transport and motility in the mouse oviduct: observation in situ. Biology of Reproduction 36 203-210

Suarez SS, Drost M, Redfern K and Gottlieb W (1990) Sperm motility in the oviduct. In Fertilization in Mammals pp 111-124 Eds BD Bavister, J Cummins and ERS Roldan. Serono Symposia, Norwell

US Department of Health and Human Services (1985) NIH Publication No. 86-23

Zhu J, Barratt CLR, Lippes J, Pacey AA, Lenton EA and Cooke ID (1994) Human oviductal fluid prolongs sperm survival Fertility and Sterility 61 360-366

Zigmond SH (1977) Ability of polymorphonuclear leukocytes to orient in gradients of chemotactic factors Journal of Cell Biology 75 606-616 